\title{
Pediatric management challenges of hyperglycemic hyperosmolar state: case series of Korean adole- scents with type 2 diabetes
}

\author{
Sumin Lee ${ }^{1}$, \\ Sukdong Yoo', \\ Ju Young Yoon', \\ Chong Kun Cheon ${ }^{1,2}$, \\ Young A Kim ${ }^{1,2}$
}

${ }^{1}$ Department of Pediatrics, Pusan National University Children's Hospital, Yangsan, Korea

${ }^{2}$ Research Institute for Convergence of Biomedical Science and Technology, Pusan National University Yangsan Hospital, Yangsan, Korea
Received: 4 May, 2021

Accepted: 22 June, 2021

Address for correspondence: Young A Kim

Department of Pediatrics, Pusan National University Children's Hospital, 20 Geumo-ro, Mulgeumeup, Yangsan 50612, Korea Email:youngflo@hanmail.net https://orcid.org/0000-0002-83325200
The hyperglycemic hyperosmolar state (HHS) is considered the most fatal complication of type 2 diabetes mellitus (DM). The number of case reports describing pediatric HHS has increased recently in parallel with obesity and the prevalence of type $2 \mathrm{DM}$ in pediatric patients. In this study, we investigated the patient characteristics and outcomes of HHS in 9 adolescents with obesity and type 2 DM. Almost all patients exhibited mixed clinical features of HHS and diabetic ketoacidosis (DKA), including characteristics such as hyperosmolality and ketoacidosis. These features made definitive diagnosis difficult; 5 out of 9 patients were initially diagnosed with DKA and were treated accordingly. Patients who were initially diagnosed with HHS received a more vigorous and appropriate fluid replacement than other patients did. No patients died, although 3 exhibited complications, such as arrhythmia, acute kidney injury requiring renal replacement therapy, rhabdomyolysis, and acute pancreatitis. Hyperosmolality with consequent severe dehydration is considered a significant factor contributing to the outcomes of patients with HHS. Therefore, early recognition of hyperosmolality is crucial for an appropriate diagnosis and adequate fluid rehydration to restore perfusion in the early period of treatment to improve patient outcomes for this rare but serious emerging condition in pediatric patients.

Keywords: Hyperglycemic hyperosmolar state, Pediatrics, Type 2 diabetes mellitus, Hyperosmolality, Ketoacidosis

\section{Highlights}

\begin{abstract}
Early recognition of hyperosmolality and appropriate fluid rehydration during the initial stages of treatment are essential for improving patient outcomes in cases of hyperglycemic hyperosmolar state, a rare but serious condition in pediatric patients.
\end{abstract}

\section{Introduction}

Hyperglycemic hyperosmolar state (HHS), which is characterized by extreme hyperglycemia and hyperosmolality without significant ketosis, is rarely observed in children, but is frequently noted in older patients with type 2 diabetes mellitus (DM) ${ }^{1-3)}$ However, in the last 2 decades, reports on HHS in pediatric patients with type 2 DM have been increasing, ${ }^{4,5)}$ and an incidence of $2 \%$ was reported by a multicenter study in the United States.6) This is probably related to the recent increase in the prevalence of pediatric obesity-related complications of type $2 \mathrm{DM}^{5,7)} \mathrm{A}$ study involving pediatric HHS cases found that 26 patients reported before 2000 were children under 9 years of age $(73 \%)$ and reported preexisting neurologic impairment and type $1 \mathrm{DM}$, whereas 71 patients in 2001-2008 were adolescents and had associated obesity and type 2 
DM. ${ }^{5)}$

This trend pertaining to pediatric HHS in Korea is not an exception and is supported by the reportedly increasing prevalence of pediatric obesity from $8.7 \%$ in 2007 to $15.0 \%$ in $2017,{ }^{8}$ although certain ethnicities (including AfricanAmerican, Caucasian, and Hispanic) seem to be more strongly associated with pediatric HHS. ${ }^{5,6,9)}$ Moreover, a pediatric study of a regional Korean population at a single center in 2009 reported that $20 \%$ of newly diagnosed diabetes patients had type $2 \mathrm{DM}$, and $53 \%$ of cases were associated with overweight or obesity. ${ }^{10)}$

Given the huge disparity in the incidences of DKA and HHS in pediatrics, general pediatricians are relatively unfamiliar with HHS and lack the knowledge and experience to diagnose and manage this type of hyperglycemic crisis. To improve clinical practice related to pediatric hyperglycemic crisis, we sought to understand the patient characteristics and outcomes of HHS in adolescents with obesity and type 2 DM by reviewing our cases and others reported in Korean literature.

\section{Case report}

\section{Data sources}

This retrospective study was conducted at a single tertiary care hospital. We searched for cases of acute hyperglycemic crisis with a blood glucose level $>600 \mathrm{mg} / \mathrm{dL}$, effective serum osmolality $>320 \mathrm{mOsm} / \mathrm{kg}$, altered consciousness, and aged 10-18 years with type 2 DM from the following sources: (1) the electronic medical record database of our institution, specifically data of patients hospitalized between January 2011 and December 2020, and (2) KoreaMed (https://koreamed.org), a service provided by the Korean Association of Medical Journal Editors. Type 2 DM was distinguished from type 1 DM based on the absence of diabetic autoantibodies and discontinuation of insulin therapy at a follow-up.

\section{Patients and clinical presentation}

Nine cases ( 5 from our hospital and 4 from the literature) were found and are summarized in Table $1{ }^{11-13)}$ All patients were previously healthy and newly diagnosed with type $2 \mathrm{DM}$ with current hyperglycemic crises. They were all obese (body mass index $>25 \mathrm{~kg} / \mathrm{m}^{2}$ ). Their body weight loss ranged from $7.5 \%$ to $18.9 \%$, which is compatible with a severe degree of dehydration. Patients arrived with altered mental status, including 2 in a coma. Patient 4 showed hypotension, which was indicative of hypovolemic shock.

\section{Diagnosis and treatment}

The cases were classified according to the diagnostic criteria for HHS stated by the International Society for Pediatric and Adolescent Diabetes (ISPAD) diagnostic criteria for HHS (Table 2)..$^{14)}$ Patients with overlapping diagnostic criteria of HHS and DKA were classified as "mixed HHS and DKA."

Five of the 9 patients had a primary diagnosis of DKA in the Emergency Department. Patient 4 was initially diagnosed with DKA, which was changed to HHS after the patient exhibited negative ketonuria (Table 3), whereas the remaining 4 patients'

Table 1. A summary of patient characteristics at the time of admission and clinical course of adolescents with HHS and type 2 diabetes

\begin{tabular}{|c|c|c|c|c|c|c|c|c|c|}
\hline \multirow{3}{*}{ Characteristic } & \multicolumn{9}{|c|}{ Patient number } \\
\hline & \multicolumn{5}{|c|}{ Our cases } & \multicolumn{4}{|c|}{ Cases from literature } \\
\hline & 1 & 2 & 3 & 4 & 5 & $6^{111}$ & $7^{11)}$ & $8^{12)}$ & $9^{13)}$ \\
\hline Age (yr) & 14 & 15 & 14 & 13 & 16 & 13 & 11 & 14 & 15 \\
\hline Sex & Male & Male & Male & Female & Male & Female & Male & Female & Male \\
\hline Height (cm) & 176 & 169 & 182 & 155 & 172 & 172 & 164 & 153 & 169 \\
\hline Weight (kg) & 74 & 69 & 80 & 66 & 101 & 86 & 70 & 82 & 85 \\
\hline Weight loss, kg (\%) & $6(7.5)$ & $8(10.4)$ & $7(8.0)$ & $9(12.7)$ & $12(10.2)$ & $20(18.9)$ & $8(10.3)$ & $8(8.9)$ & $15(15)$ \\
\hline $\mathrm{BMI}^{\dagger}, \mathrm{kg} / \mathrm{m}^{2}$ (z-score) & $26(>2)$ & $27(>2)$ & $26(>2)$ & $30(>2)$ & $40(>3)$ & $36(>3)$ & $29(>3)$ & $38(>2)$ & $35(>3)$ \\
\hline $\begin{array}{l}\text { Family history of type } 2 \\
\text { diabetes }\end{array}$ & + & + & + & None & None & None & + & + & + \\
\hline Blood pressure $(\mathrm{mmHg})$ & $100 / 60$ & $130 / 70$ & $130 / 80$ & $70 / 40$ & $90 / 60$ & $115 / 89$ & $140 / 82$ & $160 / 80$ & $145 / 83$ \\
\hline Pulse rate (beats/min) & 130 & 147 & 124 & 110 & 134 & 108 & 142 & 180 & 124 \\
\hline Mental status & Drowsy & Drowsy & Drowsy & Coma & Stupor & Confused & Confused & Coma & Drowsy \\
\hline $\begin{array}{l}\text { Time of recovery of mental } \\
\text { status (hr) }\end{array}$ & 13 & 16 & 22 & 131 & 52 & $\begin{array}{l}\text { On the 4th } \\
\text { day }\end{array}$ & 36 & On the 4th day & 26 \\
\hline Diagnosis during treatment & DKA & DKA & DKA & DKA & $\mathrm{HHS}$ & HHS & HHS & DKA & $\begin{array}{l}\text { Mixed HHS } \\
\text { and DKA }\end{array}$ \\
\hline $\begin{array}{l}\text { Diagnosis by diagnostic } \\
\text { criteria of current study }\end{array}$ & $\begin{array}{l}\text { Mixed HHS } \\
\text { and DKA }\end{array}$ & $\begin{array}{l}\text { Mixed HHS } \\
\text { and DKA }\end{array}$ & $\begin{array}{l}\text { Mixed HHS } \\
\text { and DKA }\end{array}$ & HHS & $\begin{array}{l}\text { Mixed HHS } \\
\text { and DKA }\end{array}$ & $\begin{array}{l}\text { Mixed HHS } \\
\text { and DKA }\end{array}$ & $\begin{array}{l}\text { Mixed HHS } \\
\text { and DKA }\end{array}$ & $\begin{array}{l}\text { Mixed HHS and } \\
\text { DKA }\end{array}$ & $\begin{array}{l}\text { Mixed HHS } \\
\text { and DKA }\end{array}$ \\
\hline
\end{tabular}

HHS, hyperglycemic hyperosmolar state; BMI, body mass index; and DKA, diabetic ketoacidosis.

"KoreaMed (https://koreamed.org), a service of the Korean Association of Medical Journal Editors. ${ }^{\dagger}$ Calculated with respect to the original body weight. 
Table 2. Diagnostic criteria of hyperglycemic crisis in this study

\begin{tabular}{lccc}
\hline Variable & HHS & DKA & Mixed HHS and DKA \\
\hline Blood glucose $(\mathrm{mg} / \mathrm{dL})$ & $>600$ & $>200$ & $>600$ \\
Venous pH & $>7.25 ;$ arterial $>7.30$ & $<7.3$ & $<7.3$ \\
Bicarbonate $(\mathrm{mEq} / \mathrm{L})$ & $>15$ & $<15$ & $<15$ \\
Effective osmolality $(\mathrm{mOsm} / \mathrm{kg})$ & $>320$ & $<320$ & $>320$ \\
Urine ketone & Absent or small & Significant & Significant \\
\hline
\end{tabular}

HHS, hyperglycemic hyperosmolar state; DKA, diabetic ketoacidosis.

HHS and DKA diagnoses are based on the International Society for Pediatric and Adolescent Diabetes criteria.

Table 3. A summary of the initial laboratory findings of adolescents with HHS and type 2 diabetes

\begin{tabular}{|c|c|c|c|c|c|c|c|c|c|c|}
\hline \multirow{3}{*}{ Variable } & \multicolumn{9}{|c|}{ Patient number } & \multirow{3}{*}{$\begin{array}{l}\text { Reference } \\
\text { range }\end{array}$} \\
\hline & \multicolumn{5}{|c|}{ Our cases } & \multicolumn{4}{|c|}{ Cases from literature $^{*}$} & \\
\hline & 1 & 2 & 3 & 4 & 5 & $6^{111}$ & $7^{11)}$ & $8^{12)}$ & $9^{13)}$ & \\
\hline Blood glucose (mg/dL) & 1,466 & 722 & $>700^{\dagger}$ & 1,956 & 1,250 & 1,740 & 1,140 & 1,231 & $>1,200^{\dagger}$ & $74-106$ \\
\hline Serum osmolality (mOsm/kg) & 435 & 369 & 375 & 484 & 412 & 345 & 353 & 398 & 391 & $275-295$ \\
\hline Effective osmolality ${ }^{\ddagger}(\mathrm{mOsm} / \mathrm{kg})$ & 399 & 335 & 341 & 464 & 379 & 399 & 388 & 436 & 379 & $275-295$ \\
\hline $\mathrm{pH}$ & 7.05 & 7.06 & 6.97 & 6.93 & 6.81 & 7.11 & 7.08 & 6.94 & 7.19 & $7.35-7.45$ \\
\hline Bicarbonate (mEq/L) & 7.1 & 10.8 & 2.7 & 8.8 & 5.1 & 5.6 & 3.3 & 4.7 & 6.9 & $20-28$ \\
\hline Anion gap (mEq/L) & 29 & 26 & 36 & 39 & 37 & - & - & 39.3 & 35.1 & $7-16$ \\
\hline Lactic acid (mmol/L) & 1.4 & - & 1.3 & 6.3 & 1.4 & - & - & - & - & $0.1-1.2$ \\
\hline Sodium (mEq/L) & 136 & 137 & 141 & 147 & 136 & 124 & 145 & 165 & 138 & $136-146$ \\
\hline Corrected sodium $^{\S}(\mathrm{mEq} / \mathrm{L})$ & 159 & 147 & $>151$ & 178 & 155 & 151 & 143 & 184 & $>156$ & \\
\hline BUN (mg/dL) & 68.8 & 28.2 & 35.9 & 112.3 & 55.3 & 31 & 26 & 61.6 & 44.5 & $6.6-23.6$ \\
\hline Creatinine (mg/dL) & 2.8 & 1.3 & 1.61 & 5.59 & 3.88 & 2.0 & 1.0 & 2.3 & 1.8 & $0.67-1.17$ \\
\hline $\mathrm{HbA1c}(\%)$ & 9.9 & 11.5 & 11.5 & 10.4 & 9.8 & 15.4 & 13.2 & 10.8 & 11.4 & 4-6 \\
\hline Serum insulin $(\mu \mathrm{U} / \mathrm{mL})$ & 0.7 & 3.3 & 3.5 & 7.1 & 6.6 & 1.6 & 4.1 & 1.5 & - & $0-30$ \\
\hline Serum C-peptide $(\mu \mathrm{U} / \mathrm{mL})$ & 0.7 & 1.5 & 0.9 & 4.2 & 2.7 & 6.3 & 2.5 & 3.3 & 2.5 & $0.8-4.2$ \\
\hline $\begin{array}{l}\text { Serum } \beta \text {-hydroxybutyric acid } \\
(\mathrm{mmol} / \mathrm{L})\end{array}$ & 2.77 & 2.35 & 4.16 & 0.38 & - & - & - & - & - & $0.05-0.30$ \\
\hline Urine ketone & $2+$ & $3+$ & $3+$ & Negative & $2+$ & $2+$ & $3+$ & $2+$ & $3+$ & Negative \\
\hline
\end{tabular}

$\mathrm{HHS}$, hyperglycemic hyperosmolar state; BUN, blood urea nitrogen; and HbA1c, glycosylated hemoglobin.

"KoreaMed (https://koreamed.org), a service of the Korean Association of Medical Journal Editors. ${ }^{\dagger}$ The values have exceeded the laboratory upper limit. ${ }^{\ddagger}$ Effective osmolality $=(2 \times$ corrected sodium $)+$ blood glucose $/ 18 .{ }^{~}{ }^{C}$ Corrected sodium $=$ sodium $(\mathrm{mEq} / \mathrm{L})+1.65 \times(\mathrm{blood}$ glucose $[\mathrm{mg} / \mathrm{dL}]-100) / 100$.

diagnoses were unchanged. None of the patients were an exact match with the HHS criteria; only patient 4 was diagnosed with HHS accompanied by lactic acidosis and metabolic acidosis. Eight patients exhibited characteristics of both hyperosmolality and ketoacidosis; the identification was based on significant ketonuria concurrent with high anion gap metabolic acidosis. These patients received a final diagnosis of mixed HHS and DKA.

In our cases, the patients who were initially considered to have DKA were treated according to our protocol based on the ISPAD guidelines and its update: fluid replacement for deficits over 36-48 hours with insulin infusion therapy, gradual correction of hyperglycemia with a target blood glucose level of $250-300 \mathrm{mg} / \mathrm{dL}$, and potassium and phosphorus replacement. Table 4 shows the estimated fluid requirement and actual replaced fluid volume and urine output, and Fig. 1A demonstrates the proportion of fluid quantity administered among actual patients replaced fluid volume over time. During the first 12 hours of treatment, patient 5 , who was initially thought to have HHS, received massive fluid replacement $(110 \mathrm{~mL} / \mathrm{kg})$ based on heart rate, and $63 \%$ of the required fluid volume was replaced, whereas the other 4 patients received approximately $40 \%$ of the required volume. Patient 4 required vasopressors and massive fluid replacement $(92 \mathrm{~mL} / \mathrm{kg} / 12$ hr) until sufficient resuscitation. From the beginning of the treatment, insulin was infused ranging $025-0.1 \mathrm{unit} / \mathrm{kg} / \mathrm{hr}$ in our cases. As seen in Fig. 1B and C, blood glucose and serum osmolality rapidly declined during the first 6 hours of the treatment, which slowed over 24 hours.

\section{Complications and clinical follow-up}

All patients were admitted to the intensive care unit. Although none of them died, 3 patients had acute complications during treatment. Two patients (patients 4 and 8 ) had cardiac arrhythmias and received mechanical ventilation. Patient 5 had acute pancreatitis as confirmed by abdominal computed tomography. Although almost all patients exhibited high 
Table 4. Estimated fluid requirement and actual fluid volume replaced during 48 hours of management in patients at our hospital

\begin{tabular}{lccccc}
\hline \multirow{2}{*}{ Variable } & \multicolumn{5}{c}{ Patient number } \\
\cline { 2 - 6 } & 1 & 2 & 3 & 4 & 5 \\
\hline Estimated deficit ${ }^{*}(\mathrm{~mL})$ & 6,000 & 8,000 & 7,000 & 9,000 & 12,000 \\
Estimated fluid requirement for 48 $\mathrm{hr}^{\dagger}(\mathrm{mL})$ & 11,600 & 13,250 & 13,000 & 14,000 & 17600 \\
Replaced fluid for 48 $\mathrm{hr}(\mathrm{mL})$ & 8,700 & 11,000 & 11,000 & 15,800 & 17,200 \\
Urine output/48 hr, $\mathrm{mL}(\mathrm{mL} / \mathrm{kg} / \mathrm{hr})$ & $6,670(1.9)$ & $8,580(2.4)$ & $7,590(2.1)$ & $2,680(0.8)$ & $5,100(1.4)$ \\
\hline
\end{tabular}

"Body weight loss $(\mathrm{kg}) \times 1,000 \mathrm{~mL} .{ }^{\dagger}$ Estimated deficit $(\mathrm{mL})+2 \times$ maintenance fluid $\left(\mathrm{mL}, 1,500 \mathrm{~mL} /\right.$ day $\times$ body surface area $\left.\left[\mathrm{m}^{2}\right]\right)$

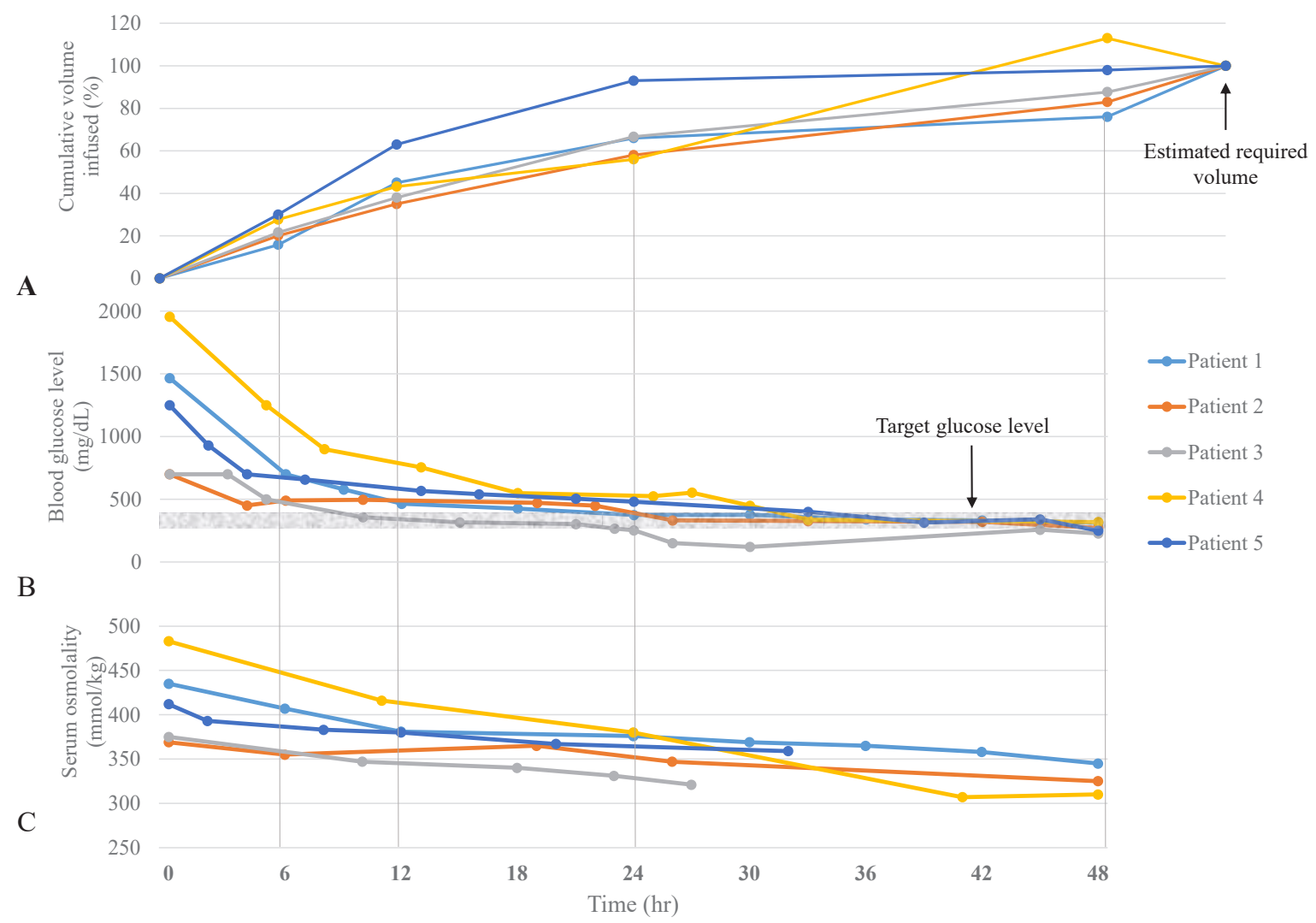

Fig. 1. The time course of the proportion of fluid quantity administered among actual replaced fluid volume (A) laboratory findings, including blood glucose level (B), serum osmolality (C), and for 48 hours of treatment.

levels of blood urea nitrogen and creatinine, and only patient 4 received renal replacement therapy initiated at 24 hours of starting treatment, whereas the others were relieved via fluid therapy. Patient 4 had several complications: (1) sudden cardiac arrest related to arrhythmia and recovery after 15 minutes of cardiopulmonary resuscitation on arrival, (2) acute kidney injury requiring renal replacement therapy for 28 days, (3) rhabdomyolysis, and (4) life-threatening rectal bleeding requiring surgical bleeding control.

All patients were negative for antibodies, including antiinsulin antibodies, islet cell antibodies, and antiglutamic acid decarboxylase antibodies. Table 1 shows the serum insulin and C-peptide levels of the patients at the time of admission. Serum insulin levels were increased in patients $2(113 \mu \mathrm{U} / \mathrm{mL})$ and 3 $(140 \mu \mathrm{U} / \mathrm{mL})$ at the 6 -month follow-up. In patient 1 , the urine C-peptide level was $0.86 \mu \mathrm{g} /$ day (reference range, $2-260$ ) at the time of admission and $43.1 \mu \mathrm{g} /$ day at the 5-month follow-up. In the initial few months after type $2 \mathrm{DM}$ diagnosis, 7 patients (patients 1-7) were treated with a combination of insulin and metformin, although patients 8 and 9 had no records of their medication. At the 6-month follow-up, 5 patients (patients 1-5) were treated with metformin, and patients 6 and 7 with lifestyle modification alone.

\section{Ethical statement}

The study was approved by the Institutional Review Board of Pusan National University Yangsan Hospital, and informed consent was waived (approval No. 05-2021-047). 


\section{Discussion}

In children with HHS, the cause of death has been reported to be multiorgan failure, such as renal failure, rhabdomyolysis, thromboembolic complications, and arrhythmia, which is thought to be related to severe dehydration, whereas other causes of death included pancreatitis and cerebral edema. ${ }^{1,5,15)}$ HHS causes greater mortality and complications than DKA does following severe dehydration. ${ }^{1,3)}$ Hence, diagnostic accuracy is essential for optimizing treatment to achieve good patient outcomes.

In this study, none of the patients met the diagnostic criteria for HHS. Patient 4 was the only patient diagnosed with HHS; the others presented mixed clinical features of HHS and DKA. Overlapping features of HHS and DKA could occur due to the accompanying hypoperfusion or lactic acidosis. ${ }^{14)}$ However, this was insufficient to explain the 8 patients with ketoacidosis, which is comparable to DKA. In the literature, we found that mixed HHS and DKA have been diagnosed during hyperglycemic crises in $14 \%$ of children and $12 \%-30 \%$ of adults. $^{15-18)}$

Although the diagnostic criteria for HHS and DKA are distinguishable due to differences in their pathogenesis, ${ }^{1,14}$ the basic mechanism of the reduced action of insulin (insulin deficiency in DKA and insulin resistance in HHS) and the consequential increase in the levels of circulating counterregulatory hormones is a pathophysiology common to both these conditions. Accordingly, clinical features frequently overlap and can lead to confusion during diagnosis. Some authors have suggested that temporary insulin deficiency is caused by $\beta$-cell exhaustion due to glucotoxicity in HHS. ${ }^{2)}$ In the case of patient 1, urine fasting $\mathrm{C}$-peptide levels were lowest at the time of admission and normalized later. Transient profound insulin deficiency was presumed to have contributed to the development of mixed HHS and DKA. Further research is required for a better understanding of the pathophysiology of mixed HHS and DKA.

Five of the 9 patients in this study were primarily diagnosed with DKA and treated accordingly. Hyperosmolality of the patients indicating HHS was overlooked, and this might be because the incidence of DKA is overwhelmingly higher than that of HHS in pediatrics. Some studies also reported similar findings of pediatric patients with HHS being incorrectly diagnosed with DKA via coding. ${ }^{9,15)}$ In HHS or mixed HHS and DKA, several studies have pointed to hyperosmolality and consequent profound hypovolemia as a major factor that influences outcomes, including altered mental status and complications such as acute kidney injury. ${ }^{15-18)}$ As observed in patients 4 and 8 , who arrived in a comatose state, had significantly higher effective osmolalities than other patients did at over $400 \mathrm{mOsm} / \mathrm{kg}$. These results suggest that hyperosmolality should be emphasized in the diagnosis of patients with hyperglycemic crisis and highlighted as an important factor during treatment.

Agrawal et al. ${ }^{15)}$ reported that patients coded incorrectly had a 3 -fold greater risk of developing complications and that suboptimal fluid administration possibly led to the increased rate of complications. Canarie et al. ${ }^{9)}$ also demonstrated that serious complications occur at $<40 \mathrm{~mL} / \mathrm{kg}$ during the first 6 hours of treatment of HHS in youth. Several studies supported that inadequate fluid resuscitation is associated with complications and poor outcomes. ${ }^{19)}$

Because the principle for managing DKA and HHS is not particularly different, ${ }^{14)}$ most patients with mild HHS may respond well to DKA treatment, as patients 1,2, and 3 did. However, patient 4, who appeared to have the most fatal outcome among our cases, received fluid therapy more actively after being rediagnosed with HHS, whereas patient 5 received massive fluid resuscitation from the start of treatment. None of the patients in this study died; we believe that adequate massive fluid resuscitation during the early hours of treatment contributed to this outcome.

Although there is no consensus on the treatment of pediatric HHS, Zeitler et al. ${ }^{1)}$ suggested vigorous fluid administration of an initial bolus of $20-\mathrm{mL} / \mathrm{kg}$ isotonic saline and repeated boluses until peripheral perfusion is established. If osmotic diuresis persists, dehydration intensifies, resulting in increased volume loss; therefore, ongoing losses also need to be replaced. ${ }^{1,14,19)}$ This does not differ from the recommendation for adults with HHS with severe hypovolemia, which includes administration of 1 $\mathrm{L} / \mathrm{hr}$ isotonic saline. ${ }^{19,20)}$ The adult protocol seems to be more appropriate for adolescents with HHS, considering their height and weight.

Insulin therapy is essential to suppress ketogenesis and hepatic gluconeogenesis. However, in HHS, insulin administration with inadequate fluid replacement expedites vascular collapse by transporting glucose and water from the extracellular fluid to the intracellular fluid. ${ }^{1)}$ Therefore, early insulin administration is unnecessary in HHS; however, in patients with mixed HHS and DKA, insulin infusion should be initiated earlier to treat ketoacidosis. ${ }^{1,14,19,20)}$ Regardless of intent, all patients at our hospital received insulin therapy in the early hours of treatment.

In conclusion, we presented the cases of 9 Korean adolescents with type 2 DM complicated with HHS or mixed HHS and DKA. Despite the limitations of this retrospective observational case study, we provide important points of diagnosis and management for this rare but serious emerging condition in pediatrics. We suggest that early recognition of hyperosmolality is essential for managing this type of hyperglycemic crisis. The focus of treatment should be on adequate fluid rehydration to restore perfusion in the early period of treatment to improve patient outcomes. An international consensus on diagnostic criteria of mixed HHS and DKA is necessary, and prospective studies are required to optimize treatment plans and improve outcomes in pediatric HHS.

\section{Notes}

Conflicts of interest: No potential conflict of interest relevant to this article was reported. 
Funding: This study received no specific grant from any funding agency in the public, commercial, or not-for-profit sectors.

Acknowledgements: This work was supported by a 2-Year Research Grant of Pusan National University.

Author contribution: Conceptualization: CKC, YAK; Data curation: SY, YAK; Formal analysis: SL, SY, YAK; Methodology: JYY, CKC; Project administration: CKC; Visualization: JYY, YAK; Writing - original draft: SL, YAK; Writing - review \& editing: SY, JYY, CKC, YAK

\section{ORCID}

Sumin Lee: 0000-0002-7058-1141

Sukdong Yoo: 0000-0003-2365-9134

Ju Young Yoon: 0000-0002-8317-1192

Chong Kun Cheon: 0000-0002-8609-5826

Young A Kim: 0000-0002-8332-5200

\section{References}

1. Zeitler P, Haqq A, Rosenbloom A, Glaser N; Drugs and Therapeutics Committee of the Lawson Wilkins Pediatric Endocrine Society. Hyperglycemic hyperosmolar syndrome in children: pathophysiological considerations and suggested guidelines for treatment. J Pediatr 2011;158:9-14, 14.e1-2.

2. Stoner GD. Hyperosmolar hyperglycemic state. Am Fam Physician 2017;96:729-36.

3. Pasquel FJ, Umpierrez GE. Hyperosmolar hyperglycemic state: a historic review of the clinical presentation, diagnosis, and treatment. Diabetes Care 2014;37:3124-31.

4. Bagdure D, Rewers A, Campagna E, Sills MR. Epidemiology of hyperglycemic hyperosmolar syndrome in children hospitalized in USA. Pediatr Diabetes 2013;14:18-24.

5. Rosenbloom AL. Hyperglycemic hyperosmolar state: an emerging pediatric problem. J Pediatr 2010;156:180-4.

6. Klingensmith GJ, Connor CG, Ruedy KJ, Beck RW, Kollman C, Haro H, et al. Presentation of youth with type 2 diabetes in the pediatric diabetes consortium. Pediatr Diabetes 2016;17:266-73.

7. Han J, Lawlor DA, Kimm SY. Childhood obesity. Lancet 2010;375:1737-48

8. Kim JH, Moon JS. Secular trends in pediatric overweight and obesity in Korea. J Obes Metab Syndr 2020;29:12-7.

9. Canarie MF, Bogue CW, Banasiak KJ, Weinzimer SA, Tamborlane WV. Decompensated hyperglycemic hyperos- molarity without significant ketoacidosis in the adolescent and young adult population. J Pediatr Endocrinol Metab 2007;20:1115-24.

10. Hong EH, Park JS, Lee HS, Cho MH, Ko CW. Clinical characteristics and laboratory findings of children who were newly diagnosed with diabetes mellitus (from 2001 to 2008). J Korean Soc Pediatr Endocrinol 2009;14:110-5.

11. Kim TY, Ahn J, Kim HS. Hyperglycemic hyperosmolar state in children with type 2 diabetes mellitus: a report of two cases. J Korean Soc Pediatr Endocrinol 2009;14:73-7.

12. Yu J, Jin H, Ko J, Kang H. A case of severe diabetic ketoacidosis in a child with type 2 diabetes. J Korean Soc Pediatr Endocrinol 2011;16:46-50.

13. Kim JH, Choi E, Rhie YJ, Lee JH, Lee KH, Nam HK. Diabetic ketoacidosis with hyperglycemic hyperosmolar state at the onset of type 2 diabetes mellitus in an adolescent male. Soonchunhyang Med Sci 2016;22:158-62.

14. Wolfsdorf JI, Glaser N, Agus M, Fritsch M, Hanas R, Rewers A, et al. ISPAD clinical practice consensus guidelines 2018: diabetic ketoacidosis and the hyperglycemic hyperosmolar state. Pediatr Diabetes 2018;19:155-77.

15. Agrawal S, Baird GL, Quintos JB, Reinert SE, Gopalakrishnan G, Boney CM, et al. Pediatric diabetic ketoacidosis with hyperosmolarity: clinical characteristics and outcomes. Endocr Pract 2018;24:726-32.

16. Pasquel FJ, Tsegka K, Wang H, Cardona S, Galindo RJ, Fayfman M, et al. Clinical outcomes in patients with isolated or combined diabetic ketoacidosis and hyperosmolar hyperglycemic state: a retrospective, hospital-based cohort study. Diabetes Care 2020;43:349-57.

17. Schmitt J, Rahman AF, Ashraf A. Concurrent diabetic ketoacidosis with hyperosmolality and/or severe hyperglycemia in youth with type 2 diabetes. Endocrinol Diabetes Metab 2020;3:e00160.

18. Wu XY, She DM, Wang F, Guo G, Li R, Fang P, et al. Clinical profiles, outcomes and risk factors among type 2 diabetic inpatients with diabetic ketoacidosis and hyperglycemic hyperosmolar state: a hospital-based analysis over a 6-year period. BMC Endocr Disord 2020;20:182.

19. Kitabchi AE, Umpierrez GE, Miles JM, Fisher JN. Hyperglycemic crises in adult patients with diabetes. Diabetes Care 2009;32:1335-43.

20. Scott AR; Joint British Diabetes Societies (JBDS) for Inpatient Care; JBDS hyperosmolar hyperglycaemic guidelines group. Management of hyperosmolar hyperglycaemic state in adults with diabetes. Diabet Med 2015;32:714-24. 